\title{
Galactose Inhibition of the Constitutive Transport of Hexoses in Saccharomyces cerevisiae
}

\author{
JULIAN NEVADO, M^ ASUNCION NAVARRO AND CLAUDIO F. HEREDIA* \\ Instituto de Investigaciones Biomédicas del CSIC, Facultad de Medicina de la U.A.M. Arzobispo, Morcillo 4 , \\ 28029 Madrid, Spain
}

Received 15 May 1992; accepted 12 August 1992

\begin{abstract}
The relationship between the pathways of glucose and galactose utilization in Saccharomyces cerevisiae has been studied. Galactose (which is transported and phosphorylated by inducible systems) is a strong inhibitor of the utilization of glucose, fructose and mannose (which have the same constitutive transport and phosphorylation systems). Conversely, all these three hexoses inhibit the utilization of galactose, though with poor efficiency. These crossinhibitions only occur in yeast adapted to galactose or in galactose-constitutive mutants.

The efficiency of galactose as inhibitor is even greater than the efficiencies of each of the other three hexoses to inhibit the utilization of each other. Phosphorylation is not involved in the inhibition and the transport of sugars is the affected step.

The cross-inhibitions between galactose and either glucose, fructose or mannose do not implicate utilization of one hexose at the expense of the other, as it occurs in the mutual interactions between the latter three sugars. It seems that, by growing the yeast in galactose, a protein component is synthesized, or alternatively modified, that once bound to either galactose or any one of the other three hexoses (glucose, fructose or mannose), cross-interacts respectively with the constitutive or the inducible transport systems, impairing their function.
\end{abstract}

KEY WORDS - Yeast; hexose transport; galactose inhibition; glycolysis; Saccharomyces cerevisiae.

\section{INTRODUCTION}

The hexose transport and phosphorylation systems in yeast have strict requirements for spacial orientation of the atoms around the atom of carbon 4 (Heredia et al., 1968). Glucose, fructose and mannose share the same transport and phosphorylation systems (Heredia et al., 1968; Kotyk, 1967; Cirillo, 1968a; Bisson and Fraenkel, 1983a), while galactose requires the additional induction of a permease (Cirillo, 1968b; Douglas and Condie, 1954) plus the enzymes of the Leloir pathway: galactokinase (EC 2.7.1.1.6; Alvarado, 1960; Schell and Wilson, 1977), galactose 1-phosphate uridyltransferase (EC 2.7.7.12; Segawa and Fukasawa, 1979) and uridine diphosphoglucose 4-epimerase (EC 5.1.3.2; Fukasawa et al., 1980). Galactose enters the glycolytic pathway after being converted first to galactose-1-phosphate by the galactokinase and then to glucose-6-phosphate by the concerted action of the transferase, epimerase and phospho-

*Addressee for correspondence.

0749-503X/93/020111-09\$09.50

() 1993 by John Wiley \& Sons Ltd glucomutase (EC 2.7.5.1). The galactose pathway is subjected to complex mechanisms of regulation (Douglas and Condie, 1954; Douglas and Hawthorne, 1964; Mortimer and Hawthorne, 1966; Bassel and Mortimer, 1971; Oshima, 1982).

Studies using non-metabolizable hexose analogues seem to indicate that in Saccharomyces cerevisiae the transport systems for glucose and galactose are not completely independent. In fact, it was found (Kotyk et al., 1975) that 6-deoxyglucose was transported with much higher affinity $\left(K_{\mathrm{m}}\right.$ $3 \mathrm{~mm}$ ) by galactose- than by glucose-grown yeast cells $\left(K_{\mathrm{m}} 500 \mathrm{~mm}\right)$, although the maximal velocity was 40 times greater in the latter. Cirillo (1968b) found that the entrance of D-arabinose (a glucose analogue) was more efficiently inhibited by Larabinose (a galactose analogue) when the yeast was grown with galactose. Bisson and Fraenkel (1983a) reported that the transport of 6-deoxyglucose in galactose-grown yeast was not only inhibited by glucose, but also partially inhibited by galactose. Certain similarities between the sequences of the proteins involved in the transport of galactose and 
glucose have also been reported (Nehelin et al., 1989).

During a study on the metabolic effects of glucose analogues on hexose metabolism in S. cerevisiae, we have found that galactose is in fact a very strong inhibitor of glucose, fructose and mannose utilization. The characteristics of this unexpected effect are described in this work.

\section{MATERIALS AND METHODS}

\section{Reagents}

Enzymes, sugars and nucleotides were from Sigma Chemical Co. (St Louis, Mo) or Boehringer (Mannheim, Germany). All other reagents were of analytical grade.

\section{Strains of yeast and culture conditions}

The yeast used in this work was $S$. cerevisiae strain W303-1A provided by Dr C. Gancedo. In some experiments, other strains of $S$. cerevisiae were used: 42407 (provided by Dr R. Lagunas), PM-1 (Heredia and Sols, 1964) and GAL80 mutant (X106-3D) provided by Dr C. Gancedo.

Cells were grown aerobically in a medium containing $1 \%$ yeast extract, $1 \%$ peptone and $2 \%$ galactose as carbon source unless otherwise stated. Erlenmeyer flasks $(500 \mathrm{ml})$ containing $150 \mathrm{ml}$ of medium were inoculated with two to five drops of a preinoculum and incubated at $30^{\circ} \mathrm{C}$ overnight in a New Brunswick Scientific rotatory shaker model G-25. Cells (usually $400-700 \mathrm{mg}$ wet weight) were collected by centrifugation, washed twice with cold distilled water and suspended in cold water at a density of about $500 \mathrm{mg}$ (wet weight) per ml. A suspension containing $1 \mathrm{mg} / \mathrm{ml}$ corresponds to an absorbance of 60 Klett units in a Klett Summerson colorimeter with filter 42 .

\section{Analytical procedures}

The utilization of different hexoses was followed by incubating the amount of yeast cells indicated in each case in reaction mixtures (usually $0.5-1 \mathrm{ml}$ ) containing $50-100 \mathrm{~mm}$ Tris- $\mathrm{HCl}, \mathrm{pH} 8.5$ and the hexoses under study at the indicated concentrations. In some cases, the reaction was run at pH 3.5 with $50 \mathrm{~mm}$-potassium phosphate buffer. No differences were observed in the inhibitory effect of galactose at these two $\mathrm{pH}$ values. The reaction mixtures were incubated, in small Erlenmeyer flasks, at $30^{\circ} \mathrm{C}$ in a rotatory shaker and appropriate aliquots $(0.05-0.1 \mathrm{ml})$ were taken at 10 -min intervals for $1 \mathrm{~h}$. The yeast was sedimented by $1-$ min centrifugation in a Eppendorf microcentrifuge and the supernatant conveniently diluted for the determination of residual sugars (Bergmeyer et al., 1985). Usually aliquots starting with $0.1 \mu \mathrm{mol}$ of the hexose to be determined (zero times) were added to $1 \mathrm{ml}$ of the corresponding reaction mixture. Glucose, fructose and mannose were determined respectively by following the reduction of NADP at $340 \mathrm{~nm}$ after incubation with hexokinase (EC 2.7.1.1) and glucose-6 phosphate dehydrogenase (EC 1.1.1.49; glucose) plus glucose phosphate isomerase (EC 5.3.1.9); fructose and mannose) and phosphomannose isomerase (EC 5.3.1.8; mannose). Galactose was determined with galactose dehydrogenase (EC 1.1.1.48). No interferences in the estimation of each sugar were observed when determined in mixtures, except for galactose, fucose and 2-deoxygalactose, which cannot be determined with galactose dehydrogenase in mixtures because they are all substrates of this enzyme.

\section{RESULTS}

\section{Utilization of hexoses alone or in combination}

The results in Table 1 show the relative efficiencies of utilization of the three fermentable hexoses, sharing the same transport and phosphorylation systems, by galactose-grown yeast cells. When given separately, mannose is utilized with a maximal rate of 1 to 1.5 times greater than glucose and fructose, although the apparent affinity for mannose is lower than for glucose and fructose. When given in combination, glucose is the preferred substrate, followed by mannose.

Galactose is utilized by galactose-grown yeast at a rate roughly equal to the rate of glucose utilization. When the galactose is given in combination with either one of the other three hexoses, it strongly inhibits the utilization of these sugars. These results were unexpected since the pathway of galactose utilization involves transport and phosphorylation systems different from those of glucose, fructose or mannose. In Figure 1 it can be seen that the inhibition by galactose of the utilization of mannose is even greater than that produced by glucose. At a concentration four times lower than that of mannose, galactose completely blocks the utilization of this sugar, while glucose, even at a higher concentration, has no significant effect. These unexpected 
Table 1. Relative efficiencies of utilization of hexoses by galactosegrown yeast cells

\begin{tabular}{|c|c|c|c|c|}
\hline \multirow[b]{2}{*}{ Substrate } & \multirow{2}{*}{$\underset{(\mathrm{mM})}{K_{\mathrm{m}}}$} & \multicolumn{3}{|c|}{$\begin{array}{c}\text { Hexose utilization rates } \\
(\mu \mathrm{mol} / \mathrm{mg} \text { yeast wet weight per } \mathrm{h})\end{array}$} \\
\hline & & Glucose & Fructose & Mannose \\
\hline Glucose & $0 \cdot 7$ & 0.6 & - & - \\
\hline Fructose & $1 \cdot 5$ & - & 0.5 & $\ldots$ \\
\hline Mannose & $2 \cdot 5$ & - & - & 1 \\
\hline Glucose + fructose & - & $0 \cdot 3$ & $0 \cdot 1$ & - \\
\hline Glucose + mannose & - & $0 \cdot 4$ & - & $0 \cdot 1$ \\
\hline Fructose + mannose & - & -- & $0 \cdot 2$ & 1 \\
\hline
\end{tabular}

Reaction mixtures $(1 \mathrm{ml}$ ) containing $50 \mathrm{~mm}-\mathrm{Tris}-\mathrm{HCl}, \mathrm{pH} 8.5 ; 60 \mathrm{mg}$ (wet weight) yeast and the indicated hexoses alone $(50 \mathrm{~mm})$ or in combination $(50 \mathrm{~mm}$ each sugar) as indicated, were incubated at $30^{\circ} \mathrm{C}$ in a rotatory shaker and aliquots $(0.05$. $0.1 \mathrm{ml}$ ) were taken at 10 -min intervals for $1 \mathrm{~h}$ for the determination of the corresponding sugars as indicated in Methods. For the determination of the $K_{\mathrm{m}}$ values, $20 \mathrm{mg}$ of yeast (wet weight) and $10 \mu \mathrm{mol}$ of the corresponding hexose were incubated in reaction mixtures containing $50 \mathrm{~mm}$-Tris $-\mathrm{HCl}, \mathrm{pH} 8.5$. The hexose concentrations $(0.5-10 \mathrm{mM})$ were adjusted by varying the volumes of the reaction mixtures from 1 to $20 \mathrm{ml}$. The rates of disappearance of the hexoses were plotted (Lineweaver-Burk plot) against the hexose concentrations.

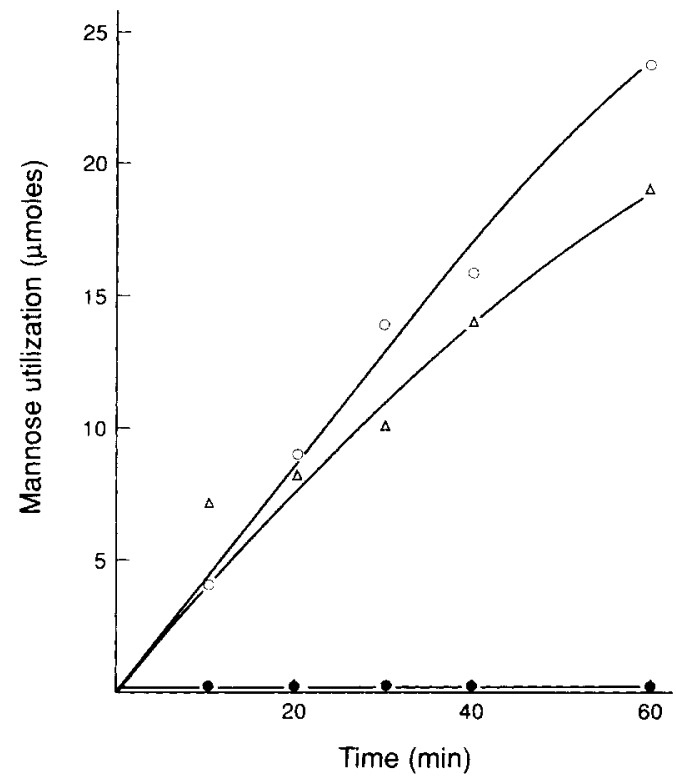

Figure 1. Differential effect of glucose and galactose on mannose utilization. Reaction mixtures $(0.5 \mathrm{ml})$ containing $100 \mathrm{~mm}$ Tris- $\mathrm{HCl}, \mathrm{pH} 8 \cdot 5,30 \mathrm{mg}$ (wet weight) yeast and mannose ( $80 \mathrm{~mm}$ ) alone $(O)$ or with $40 \mathrm{~mm}$-glucose $(\Delta)$ or $20 \mathrm{~mm}$-galactose $(\bullet)$ were incubated at $30^{\circ} \mathrm{C}$ in a rotatory shaker in small Erlenmeyer flasks. Aliquots were taken at the indicated times for determination of residual mannose. results led us to investigate more thoroughly this galactose effect.

The results of Figures 2 and 4 show that a concentration of galactose as low as $5 \mathrm{~mm}$ is enough to block completely the utilization of $80 \mathrm{~mm}$-mannose. It can also be seen in Figure 2 that as galactose disappears from the reaction mixtures, the cells start to utilize mannose, reaching rate values approaching that of the control without galactose. These results rule out the possibility that the observed inhibition is due to impurities present in the preparations of galactose. Galactose is a less efficient inhibitor of the utilization of glucose and fructose (see below).

\section{Effect of growth conditions and yeast strain on the inhibitory effect of galactose}

The inhibition by galactose of the utilization of glucose, fructose and mannose depends on the growth conditions of the cells and on the yeast strain. The results in Figure 3 show that, in contrast with the observations in the case of galactose-grown cells, inhibition does not occur when the yeast is grown with glucose as the sole carbon source. Concentrations of galactose as high as $40 \mathrm{~mm}$ have no effect on the utilization of mannose $(80 \mathrm{~mm})$. These results are in good agreement with the poor 


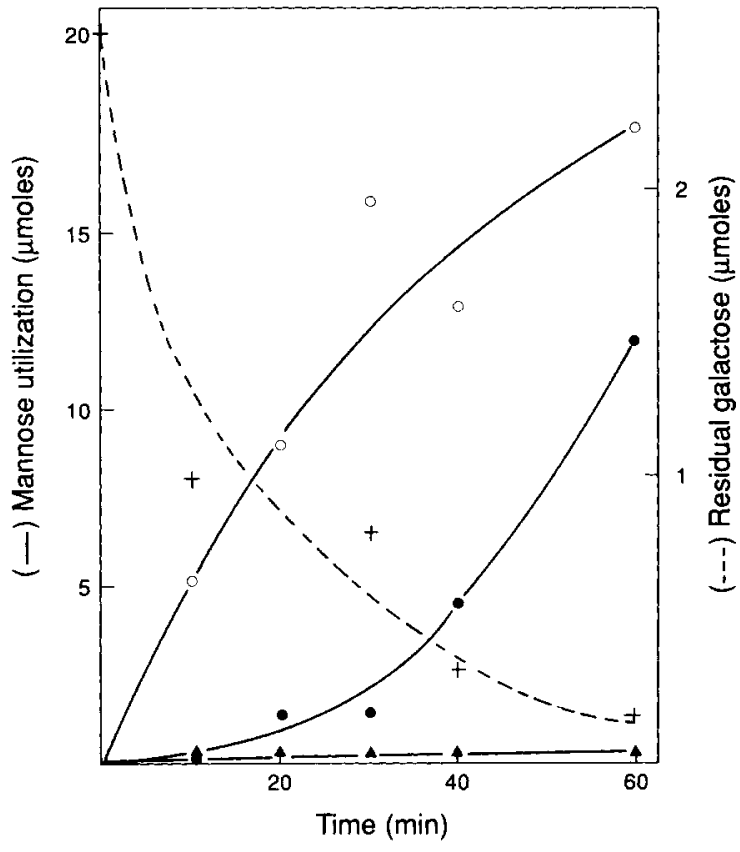

Figure 2. Effect of the concentration of galactose on the utilization of mannose. Reaction mixtures $(0.5 \mathrm{ml})$ containing $100 \mathrm{~mm}$ Tris- $\mathrm{HCl}, \mathrm{pH} 8 \cdot 5 ; 80 \mathrm{~mm}$-mannose alone $(O)$ or with $5 \mathrm{~mm}(\odot)$ or $20 \mathrm{~mm}(\Delta)$ galactose and $40 \mathrm{mg}$ (wet weight) of yeast were incubated at $30^{\circ} \mathrm{C}$ in small Erlenmeyer flasks in a rotatory shaker Aliquots were taken at the indicated intervals to determine residual mannose. The disappearance of galactose ( $5 \mathrm{~mm}$ ) (broken line) is also shown.

apparent affinity of the glucose transport system for galactose (Heredia et al., 1968).

On the other hand, the inhibitory effect of galactose is not observed in galactose-grown strain PM-1 with very low capability to utilize galactose (ca. $0.005 \mu \mathrm{mol} / \mathrm{mg}$ yeast wet weight per h). Strain 42407 , which utilizes galactose (ca. $0.5 \mu \mathrm{mol} / \mathrm{mg}$ yeast wet weight per $h$ ) as efficiently as W303-1 A, is also sensitive, but a higher concentration of galactose $(40 \mathrm{~mm})$ is required to inhibit completely the utilization of mannose $(80 \mathrm{~mm})$. Consequently, it seems that some relationship exists between the inhibitory effect of galactose and the operativity of the galactose pathway. This point has been corroborated by using a galactose-constitutive strain of yeast (X106-3D). In contrast with what occurs in the case of inducible strains, the inhibition by galactose of mannose utilization in this strain takes place not only when the cells have been grown with galactose, but also with $2 \%$ glycerol as the sole carbon source (results not shown).

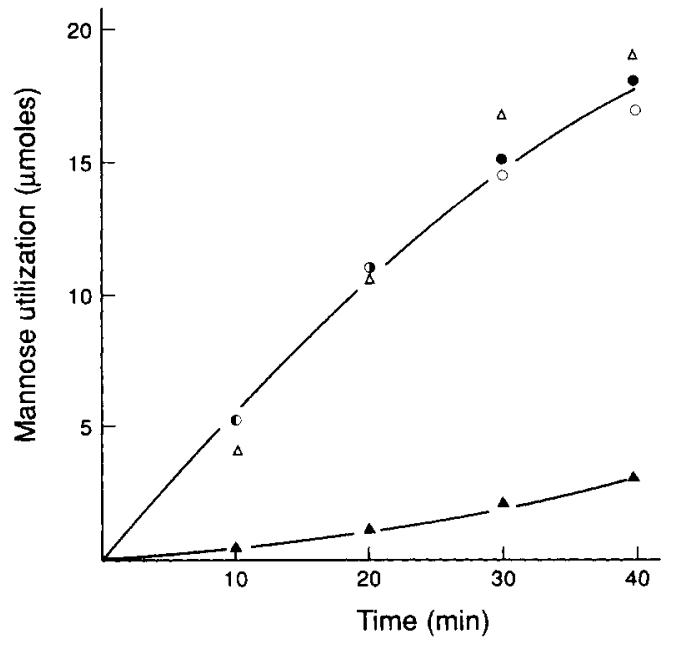

Figure 3. Effect of growth conditions on the inhibition by galactose of mannose utilization. Reaction mixtures $(0.3 \mathrm{ml})$ containing $25 \mathrm{~mm}$ - $\mathrm{Tris}-\mathrm{HCl}, \mathrm{pH} 8.5 ; 80 \mathrm{~mm}$-mannose either alone (open symbols) or with $40 \mathrm{~mm}$-galactose (filled symbols) and $40 \mathrm{mg}$ (wet weight) yeast were incubated at $30^{\circ} \mathrm{C}$ in a rotatory shaker. At the indicated times, aliquots were removed for mannose determination. Circles, yeast grown with glucose; triangles, yeast grown with galactose.

\section{Differential effect of galactose on the utilization of glucose and mannose}

Galactose is less efficient in inhibiting the utilization of glucose and fructose than that of mannose. This differential effect is illustrated in Figure 4. A concentration of $10 \mathrm{~mm}$ galactose that completely blocks the utilization of mannose has no significant effect on the utilization of glucose (or fructose, not shown) and even a greater concentration of galactose $(20 \mathrm{~mm})$ has only a limited inhibitory effect. This differential effect of galactose is not correlated with differences in the efficiency of glucose and mannose to inhibit the utilization of galactose. No significant differences are observed in the inhibition produced by the same concentration of glucose and mannose on the utilization of galactose (Figure 4).

\section{Effect of non-metabolizable galactose analogues on the utilization of mannose}

The inhibitory effect of galactose on the utilization of glucose, fructose and mannose might be a consequence of interactions between substrates and inhibitor at the level of the transport or phosphorylation systems. To distinguish between these two possibilities, the effect of D-fucose (6deoxygalactose) on the utilization of mannose was 


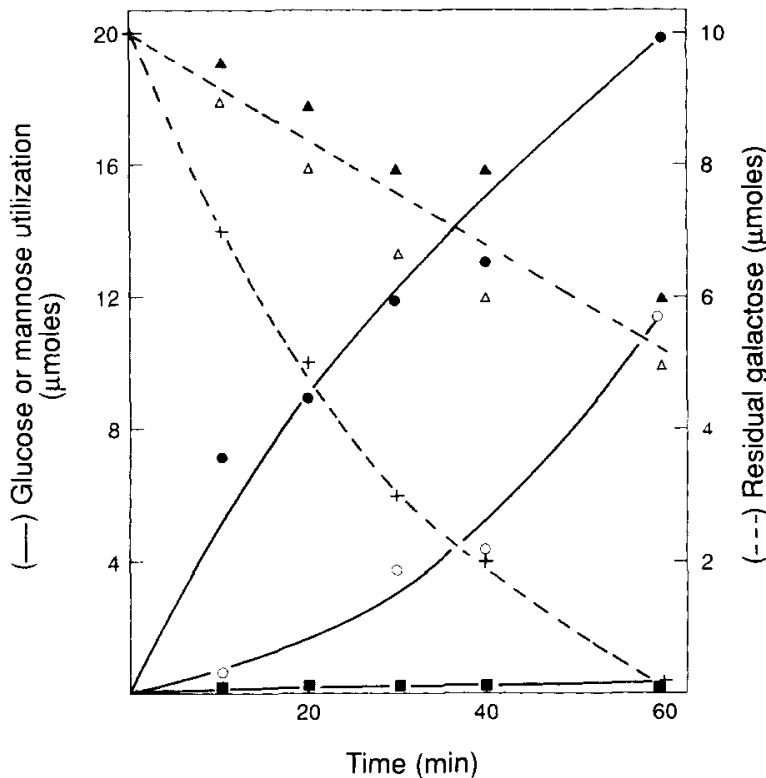

Figure 4. Differential effect of galactose on the utilization of glucose and mannose. Reaction mixtures $(1 \mathrm{ml})$ containing $50 \mathrm{~mm}-\mathrm{Tris}-\mathrm{HCl}, \mathrm{pH} 8 \cdot 5 ; 50 \mathrm{mg}$ (wet weight) yeast and $50 \mathrm{~mm}$ glucose (-) or mannose (a) plus $10 \mathrm{~mm}$-galactose or $50 \mathrm{~mm}$ glucose plus $20 \mathrm{~mm}$-galactose $(0)$, were incubated at $30^{\circ} \mathrm{C}$ in a rotatory shaker. Aliquots were taken at the indicated times for determination of hexoses. Broken lines represent utilization of $10 \mathrm{~mm}$-galactose either alone $(+)$ or in mixtures with $50 \mathrm{~mm}$ mannose $(\Delta)$ or $50 \mathrm{~mm}$-glucose $(\triangle)$. The rate of utilization of glucose alone $(50 \mathrm{~mm})$ is practically the same as that observed in the presence of $10 \mathrm{~mm}$-galactose.

tested. D-Fucose is transported by the galactose transport system, is a gratuitous inducer of this system (Cirillo 1968b) but is not phosphorylated by galactokinase (Alvarado, 1960; Schell and Wilson, 1977). The results in Figure 5 show that D-fucose inhibits the utilization of mannose, ruling out phosphorylation as being the cause of the inhibition and indicating that the step affected is the transport itself.

The inhibition by $D$-fucose requires a much higher concentration than that by galactose $(100 \mathrm{mM}$ vs $5 \mathrm{~mm}$ in the case of galactose) and preincubation of the yeast with the inhibitor is a required condition, as shown in Figure 5. These requirements could be a consequence of the poor efficiency of D-fucose to replace galactose as a structural analogue (Cirillo, $1968 \mathrm{~b}$ ). As in the case of galactose, the inhibition by D-fucose with mannose is greater than with glucose and does not occur in glucose-grown yeast. As shown in Figure 6, the utilization of both sugars is strongly inhibited during the first $20 \mathrm{~min}$, but after this time the utilization of glucose attains almost

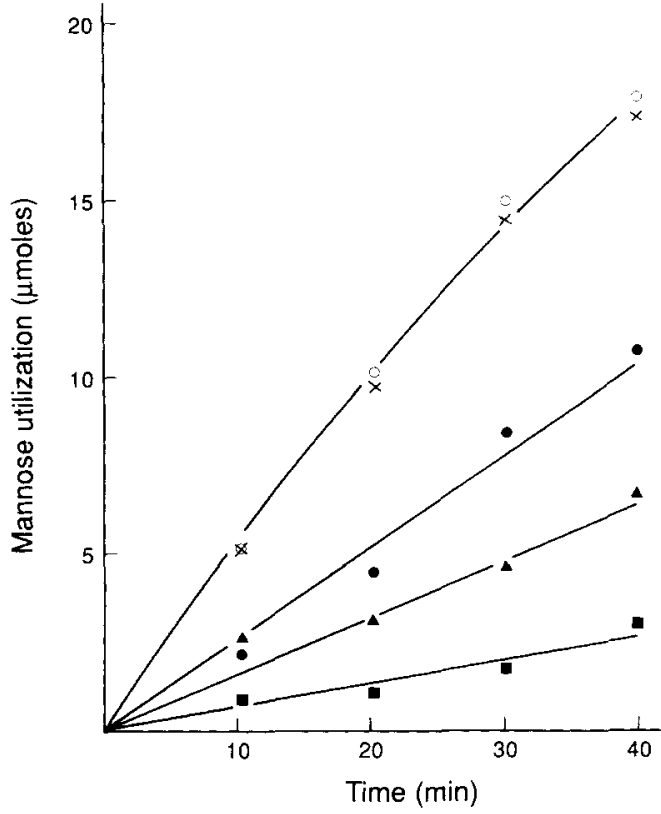

Figure 5. Effect of D-fucose on the utilization of mannose. Reaction mixtures $(0.5 \mathrm{ml})$ containing $100 \mathrm{~mm}-\mathrm{Tris}-\mathrm{HCl}$, pH 8.5; $100 \mathrm{~mm}-\mathrm{D}-$ fucose, $250 \mathrm{mg}$ (wet weight) yeast were preincubated at $30^{\circ} \mathrm{C}$ in a rotatory shaker for the indicated times. At the end of the preincubation, aliquots of $80 \mu \mathrm{l}$ were transferred to flasks containing $170 \mu \mathrm{l}$ of $150 \mathrm{~mm}$-mannose and the incubation was continued at $30^{\circ} \mathrm{C}$. Aliquots were removed at $10-\mathrm{min}$ intervals for determination of mannose. Controls without preincubation: minus $(O)$, or plus $(x) D$-fucose. Preincubation with

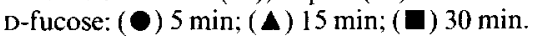

normal rates, while that of mannose remains greatly inhibited. The recovery of glucose utilization is probably a consequence of the greater efficiency of glucose to displace D-fucose from the yeast cells and indicates that the loss of hexose utilization is not due to an indiscriminate and irreversible toxic effect of $D$-fucose. Since the concentration of D-fucose required for inhibition (100 $\mathrm{mm})$ is much higher than that of galactose $(5 \mathrm{~mm})$, we have to be sure that the preparation of $\mathrm{D}$-fucose was free of galactose. We have estimated that D-fucose contains less than $0 \cdot 1 \%$ of material phosphorylable with ATP by yeast cell-free extracts of galactose-grown yeast, or reactive with galactose oxidase (EC 1.1.3.9).

The effect of 2-deoxygalactose (another nonmetabolizable galactose analogue) on the utilization of mannose is shown in Figure 7. Unlike D-fucose, once inside the cells, 2-deoxygalactose is phosphorylated by the galactokinase, although with much lower efficiency than galactose (Alvarado, 1960). The inhibition by 2-deoxygalactose is a 


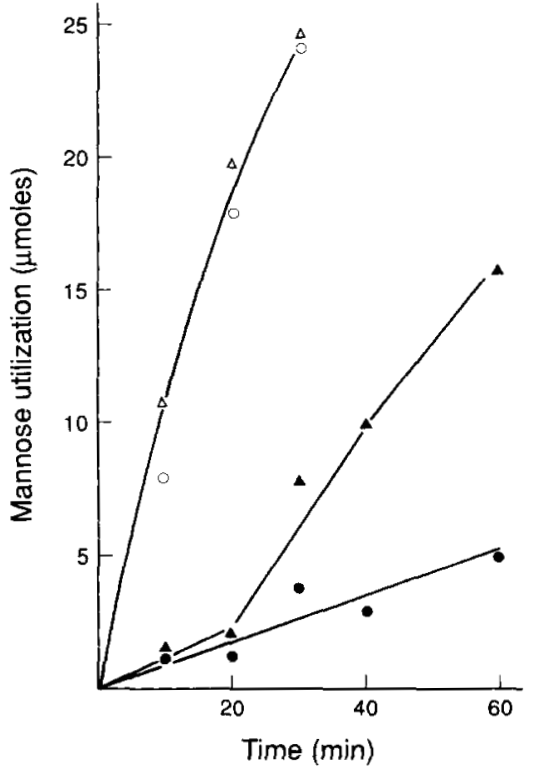

Figure 6. Differential effect of D-fucose on the utilization of mannose and glucose. Yeast cells ( $175 \mathrm{mg}$ wet weight) were preincubated at $30^{\circ} \mathrm{C}$ for $30 \mathrm{~min}$ in reaction mixtures $(0.5 \mathrm{ml})$ containing $100 \mathrm{~mm}$-D-fucose and $100 \mathrm{~mm}-\mathrm{Tris}-\mathrm{HCl}, \mathrm{pH} 8 \cdot 5$. After this preincubation, aliquots $(115 \mu 1)$ were transferred to Erlenmeyer flasks containing $135 \mu \mathrm{l}$ of $185 \mathrm{~mm}$-mannose (A) or glucose (A). Non-preincubated controls were run in parallel with the same concentrations of mannose $(O)$ or glucose $(\triangle)$. Aliquots were removed at intervals to determine the residual glucose or mannose.

two-phase process and increases as the time of incubation progresses. After $30 \mathrm{~min}$ of incubation, the utilization of mannose is only a very small fraction of the control. In line with these results, when the yeast cells are preincubated with 2-deoxygalactose for $30 \mathrm{~min}$, they are practically unable to utilize mannose. This double effect of the 2-deoxygalactose is different from that observed with galactose and D-fucose, and can be explained by an effect of the free sugar during the first minutes (as an analogue of galactose) followed by a second effect of the accumulated 2-deoxygalactose 1-phosphate and/or its derivatives (Heredia et al., 1964; Lagunas and Moreno, 1992). Galactosamine, another phosphorylable galactose analogue, behaves very like 2deoxygalactose as inhibitor of mannose utilization (results not shown).

\section{Impairment of hexose utilization in mixtures with galactose}

The cross-inhibition between galactose and either one of the other three fermentable hexoses (glucose,

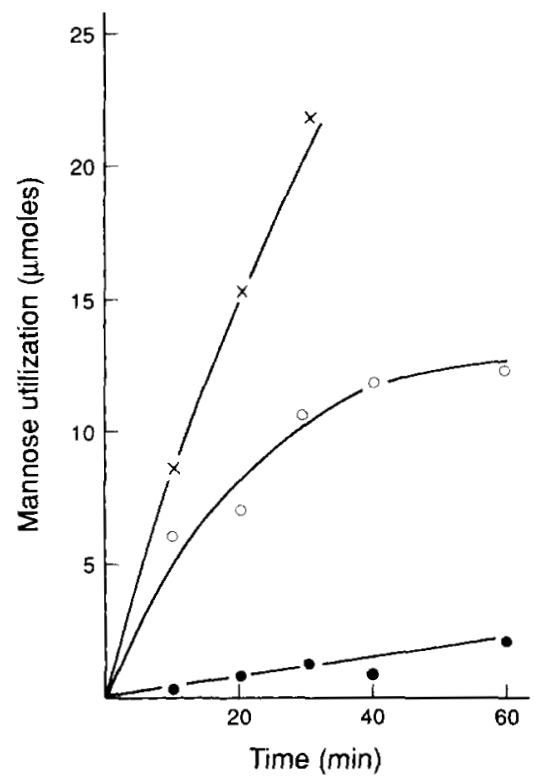

Figure 7. Effect of 2-deoxygalactose on the utilization of mannose. Reaction mixtures $(1 \mathrm{ml})$ containing $50 \mathrm{~mm}$-Tris- $\mathrm{HCl}$, $\mathrm{pH} 8.5 ; 8 \mathrm{~mm}$-2-deoxygalactose and $160 \mathrm{mg}$ (wet weight) of yeast were incubated at $30^{\circ} \mathrm{C}$ in a rotatory shaker. Aliquots of $250 \mu 1$ were transferred at zero time (open symbols) and after $30 \mathrm{~min}$ (filled symbols) to Erlenmeyer flasks containing $50 \mu 1$ of $500 \mathrm{~mm}$ mannose. Aliquots were removed at the indicated times for determination of mannose. $(x)$, Control without 2-deoxygalactose.

fructose and mannose) shown above, led us to envisage situations in which the yeast cells, with plenty of sugars at their disposal, should have severe limitations to use any of them, when given in appropriate combinations with galactose. That this is so is shown in Figure 8. When an equimolar mixture of galactose and mannose (50 $\mathrm{mm}$ each) was offered to galactose-grown yeast, the rate of total hexose consumption was very low (no more than $20 \%$ as compared with that observed in the case of mixtures of glucose and mannose (Figure 8A), in spite of the fact that the yeast utilizes mannose or galactose at normal rates when given separately (Figures 2 and 4 ). In contrast to the results found with galactose, when a mixture of glucose and mannose $(50 \mathrm{~mm})$ each was used, the rate of total hexose consumption reached values similar to those found with each sugar when given separately (ca $0.5 \mu \mathrm{mol} / \mathrm{mg}$ yeast wet weight per h). According to the results shown in Table 1, glucose is utilized in these mixtures with a higher efficiency than mannose (Figure 8B). It is important to emphasize that the observed rate of hexose utilization in mixtures of galactose and mannose is an upper value because of the difficulty of estimating a maximum of $10-20 \%$ total substrate consumption. 

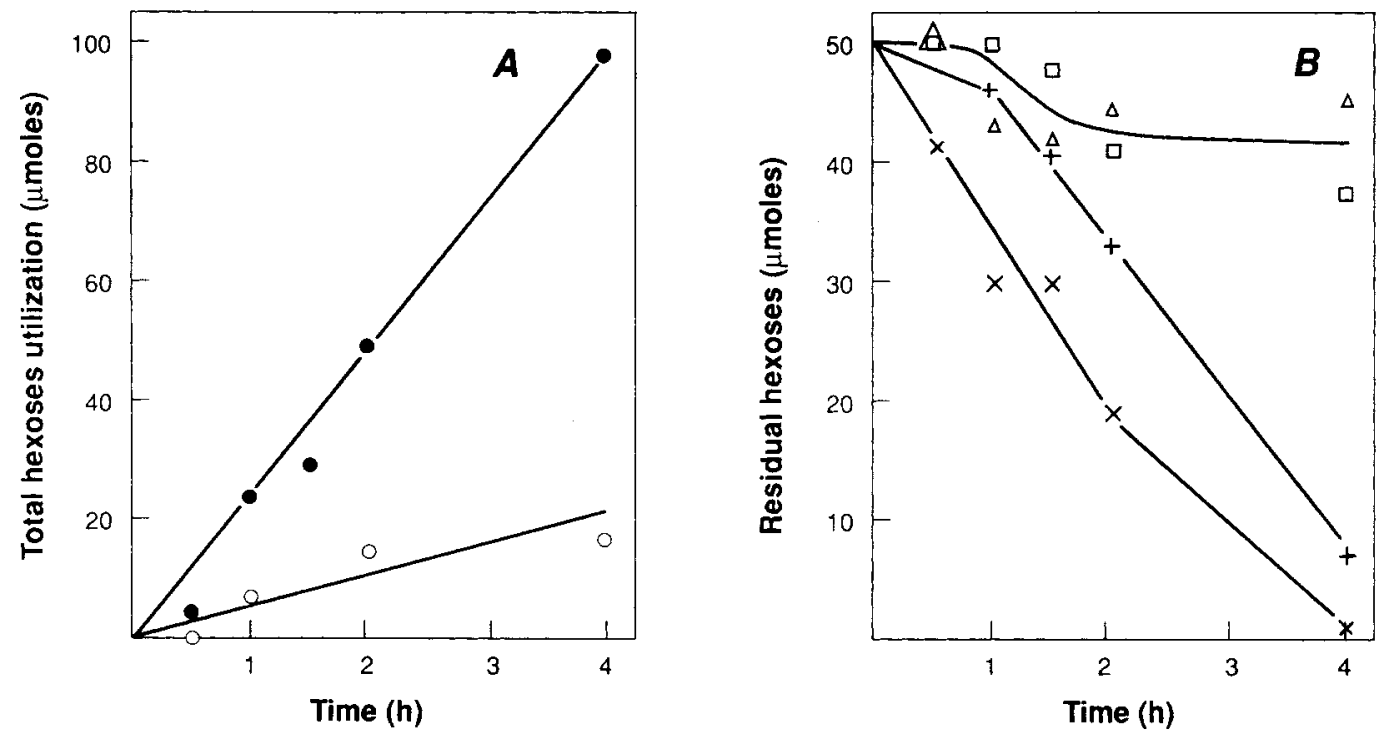

Figure 8. Impairment of hexose utilization in equimolar mixtures of galactose and mannose. Reaction mixtures (1 ml) containing: $50 \mathrm{~mm}$-Tris- $\mathrm{HCl}, \mathrm{pH} 8.5,50 \mathrm{~mm}$-mannose with $50 \mathrm{~mm}$-glucose (O) or $50 \mathrm{~mm}$-galactose $(O)$ and $50 \mathrm{mg}$ (wet weight) of yeast were incubated in a rotatory shaker. Aliquots were removed at the indicated intervals to estimate the residual hexoses. (A) Total hexose utilization. (B) Residual hexoses: $(x)$ glucose; $(+)$ mannose in mixtures with glucose; $(\square)$ galactose; $(\triangle)$ mannose in mixtures with galactose.

As mannose has lower efficiency than galactose as inhibitor (Figure 4), galactose is utilized in the mixtures, although very slowly, and in accordance with the results shown in Figure 2, the utilization of mannose is progressively less affected. After $24 \mathrm{~h}$ incubations (data not shown in Figure 8), both sugars have disappeared from the reaction mixtures.

\section{DISCUSSION}

The results presented in this paper indicate that galactose greatly interferes with the utilization of mannose, glucose and fructose by galactose-grown yeast. Galactose is much more efficient as an inhibitor of mannose utilization than glucose, which shares with mannose the same constitutive transport and phosphorylation systems (Figure 1). Conversely, the results in Figure 4 show that glucose and mannose inhibit the utilization of galactose, but their efficiency as inhibitors is much weaker than that of galactose. These results indicate that there is a relationship between the first steps of the pathways of glucose and galactose utilization in spite of their apparently different nature (Oshima, 1982; Gancedo, 1992). From the available data, it seems unlikely that these interactions would take place at the phosphorylation step, since hexokinase cannot phosphorylate galactose (Sols et al., 1958) nor can galactokinase phosphorylate glucose, fructose or mannose (Alvarado, 1960; Schell and Wilson 1977). D-Fucose, which is transported by the inducible galactose transport system (Cirillo; 1968b) but is not a substrate of galactokinase (Alvarado, 1960; Schell and Wilson, 1977), mimics the action of galactose (Figures 5 and 6 ). These results rule out phosphorylation as being involved in the observed inhibition produced by galactose and points to the transport of glucose, fructose and mannose as being the step impaired by galactose. This conclusion received further support by the facts that galactose inhibits not only the utilization but also the uptake of $\left[{ }^{14} \mathrm{C}\right]$-mannose and $\mathrm{L}$-arabinose is also an inhibitor, although less efficient than D-fucose (results not shown).

The inhibitory effect of galactose does not take place in cells non-adapted to galactose (Figure 3), indicating that it is not due to a sequestering by galactose of components peculiar to the constitutive transport system. The results in Figure 8 show that neither galactose nor mannose are significantly utilized when given in appropriate mixtures to galactose-grown yeast, although both are utilized when given separately (Figures 2 and 4 ). These results indicate that each sugar inhibits the utiliz- 
ation of the other without being itself significantly utilized. Consequently the cross-inhibitions between these two sugars cannot be explained as a result of competition for a component common to the two transport systems. If this was the case, one of the two sugars (mannose or galactose) should be utilized at the expense of the utilization of the other, as is observed when mixtures of equimolar concentrations of glucose and mannose (two sugars which share the transport system) are given to the yeast cells (Figure 8B). Rather it seems that each sugar (presumably bound to some newly synthesized protein component(s) in response to galactose) arrests the uptake of the others by interacting with their corresponding transport system. When the bound sugar is galactose, it will interfere with the transport of glucose, fructose or mannose. Conversely, when the bound sugar is any one of the other three hexoses, it will inhibit the uptake of galactose. The affinity of the protein component apparently is much higher for galactose than for the three hexoses as inferred from the fact that galactose is much more efficient as inhibitor than any one of the other hexoses (Figure 4). The higher inhibitory effect of galactose on the utilization of mannose as compared with that of glucose (Figure 4) could be the result of the lower efficiency of mannose to be transported by the constitutive transport system (Table 1).

Other non-metabolizable galactose analogues (2-deoxygalactose and galactosamine) are phosphorylated by the galactokinase (Alvarado, 1960). The inhibition of mannose utilization by these compounds progressively increased with time (Figure 7) as it corresponds to the double effect produced first by the sugar (as a galactose analogue) and later on by the intracellular accumulated sugar phosphate(s) (Heredia et al., 1964; Lagunas and Moreno, 1992).

As for the physiological meaning, the marked inhibitory effect of galactose could be a mechanism developed by the cell to circumvent the deleterious effect that even low concentrations of glucose (or mannose and fructose) have on the utilization of galactose. It is well known that these three sugars greatly impair the utilization of galactose, both by inactivating enzymes peculiar to the galactose pathway and by preventing their synthesis (Gancedo, 1992). We cannot envisage aprioristic reasons why glucose, fructose or mannose should be utilized preferentially to galactose, even when this sugar is the main carbon source. The case described in this work could be representative of instances in which galactose is preferentially used even in the presence of the other three fermentable hexoses.

\section{ACKNOWLEDGEMENTS}

This work was supported by a grant (PB87-0206) from the DGICYT, Promocion General del Conocimiento. We are grateful to Dr J. M. Gancedo and Dr A. Coloma for critical reading of the manuscript.

\section{REFERENCES}

Alvarado, F. (1960). Substrate specificity of Saccharomyces fragilis galactokinase. Biochim. Biophys. Acta. 41, 233-238.

Bassel, J. and Mortimer, R. (1971). Genetic order of the galactose structural genes in Saccharomyces cerevisiae. J. Bacteriol. 108, 179-183.

Bergmeyer, H. V., Bergmeyer, J. and Grabl, M. (Eds) (1985). Methods of Enzymatic Analysis. VCH, Verlagsgesellschaft $\mathrm{mbH}$, Weinheim.

Bisson, L. F. and Fraenkel, D. G. (1983a). Involvement of kinases in glucose and fructose uptake by Saccharomyces cerevisiae. Proc. Natl. Acad. Sci. USA 80 , 1730-1734.

Bisson, L. F. and Fraenkel, D. J. (1983b). Transport of 6deoxyglucose in Saccharomyces cerevisiae. J. Bacteriol. 155, 995-1000.

Cirillo, V. P. (1968a). Relationship between sugar structure and competition for the sugar transport system in baker's yeast. J. Bacteriol. 95, 603-611.

Cirillo, V. P. (1968b). Galactose transport in Saccharomyces cerevisiae. J. Bacteriol. 95, 1727-1731.

Douglas, H. C. and Condie, F. (1954). The genetic control of galactose utilization in Saccharomyces. J. Bacteriol. 68, 662-670.

Douglas, H. C. and Hawthorne, D. C. (1964). Enzymatic expression and genetic linkage of genes controlling galactose utilization in Saccharomyces. Genetics. 49 , 837-844.

Fukasawa, T., Obonai, K. Segawa, T. and Nogi, Y. (1980). The enzymes of the galactose cluster in Saccharomyces cerevisiae. II. Purification and characterization of uridine diphosphoglucose 4-epimerase. J. Biol. Chem. 255, 2705-2707.

Gancedo, J. M. (1992). Carbohydrate Metabolism in yeast. In Morgan, M. (Ed.), Carbohydrate Metabolism in Cultured Cells. Plenum Press, New York, pp. 245-286.

Heredia, C. F. and Sols, A. (1964). Metabolic studies with 2-deoxyhexoses. II. Resistance to 2-deoxyglucose in a yeast mutant. Biochim. Biophys. Acta 86, 224-228.

Heredia, C. F., DelaFuente, G. and Sols, A. (1964). Metabolic studies with 2-deoxyhexoses. I. Mechanisms of inhibition of growth and fermentation in baker's yeast. Biochim. Biophys. Acta 86, 216-223.

Heredia, C. F., Sols, A. and DelaFuente, G. (1968). Specificity of the constitutive hexose transport in yeast. Eur. J. Biochem. 5, 321-329.

Kotyk, A. (1967). Properties of the sugar carrier in baker's yeast. II. Specificity of transport. Folia Microbiol. 12, 121-131. 
Kotyk, A., Michaljanicova, D., Veres, K. and Soukupova, V. (1975). Transport of 4-deoxy- and 6-deoxy-glucose in baker's yeast. Folia Microbiol. 20, 496-503.

Lagunas, R. and Moreno, E. (1992). Inhibition of glycolysis by 2-deoxygalactose in Saccharomyces cerevisiae. Yeast 8, 107-115.

Mortimer, R. K. and Hawthorne, D. C. (1966). Genetic mapping in Saccharomyces. Genetics 53, 165-173.

Nehelin, J. O., Calberg, M. and Ronne, H. (1989). Yeast galactose permease is related to yeast and mammalian glucose transporters. Gene 85, 313-319.

Oshima, Y. (1982). Regulatory circuits for gene expression: The metabolism of galactose and phosphate. In Strathern, J. N., Jones, E. W. and Broach, J. R. (eds)
The Molecular Biology of the Yeast Saccharomyces. Metabolism and Gene Expression. Cold Spring Harbor Laboratory, Cold Spring Harbor, NY, pp. 159-180.

Schell, M. A. and Wilson, D. B. (1977). Purification and properties of galactose kinase from Saccharomyces cerevisiae. J. Biol. Chem. 252, 1162-1166.

Segawa, T. and Fukasawa, T. (1979). The enzymes of the galactose cluster in Saccharomyces cerevisiae. Purification and characterization of galactose-1-phosphate uridyl transferase. J. Biol. Chem. 254, 10707-10709.

Sols, A., DelaFuente, G., Villar-Palasi, C. and Asensio, C. (1958). Substrate specificity and some other properties of baker's yeast hexokinase. Biochim. Biophys. Acta 30, 92-101. 